\title{
New infrared star clusters in the southern Milky Way with 2MASS
}

\author{
C. M. Dutra ${ }^{1}$, E. Bica ${ }^{2}$, J. Soares ${ }^{2}$, and B. Barbuy ${ }^{1}$ \\ ${ }^{1}$ Universidade de São Paulo, Instituto de Astronomia, Geofísica e Ciências Atmosféricas, CP 3386, São Paulo 01060-970, \\ SP, Brazil \\ ${ }^{2}$ Universidade Federal do Rio Grande do Sul, Instituto de Física, CP 15051, Porto Alegre 91501-970, RS, Brazil
}

Received 12 November 2002 / Accepted 23 December 2002

\begin{abstract}
We carried out a 2 MASS $J, H$ and $K_{\mathrm{s}}$ survey of infrared star clusters in the Milky Way sector $230^{\circ}<\ell<350^{\circ}$. This zone was the least studied in the literature, previously including only 12 infrared clusters or stellar groups with $|b|<10^{\circ}$, according to the recent catalogue by Bica et al. (2003). We concentrated efforts on embedded clusters, which are those expected in the areas of known radio and optical nebulae. The present study provides 179 new infrared clusters and stellar groups, which are interesting targets for detailed future infrared studies. The sample of catalogued infrared clusters and stellar groups in the Galaxy is now increased by $63 \%$.
\end{abstract}

Key words. Galaxy: open clusters and associations: general - infrared: general

\section{Introduction}

The development of detectors and the creation of tools to more efficiently probe the sky in the infrared domain are producing a rapid progress in the knowledge of the related objects (e.g. Lada \& Lada 1991; Hodapp 1994; Deharveng et al. 1997). In particular, near infrared imaging surveys such as the Two Micron All Sky Survey (hereafter 2MASS, Skrutskie et al. 1997) and the Deep NIR Southern Sky Survey (DENIS; Epchtein et al. 1997) are providing material for cluster identifications. Basically these studies employed: (i) visual inspection of the 2MASS images (e.g. Dutra \& Bica 2000a; Hurt et al. 2000; Dutra \& Bica 2001; Soares \& Bica 2002), or (ii) automated searches based on stellar density contrast (e.g. Carpenter 2000; Ivanov et al. 2002; Reylé \& Robin 2002). Dutra \& Bica (2000a) scanned by eye entirely a specific area encompassing the Galactic center, whereas Dutra \& Bica (2001) searched for embedded clusters and stellar groups right in the areas of known nebulae: the method has proven to be very efficient in the Cygnus $X$ area and other parts of the sky.

Bica et al. (2003) gathered the infrared clusters and stellar groups to that date into a catalogue including 276 objects. As described therein stellar groups are less dense than clusters, but their relation to nebulae, among other evidence, suggest them to be physical systems. In that study it became clear that the Southern Milky Way was the least probed sector in the Galaxy. Recently, two additional works report IR star cluster discoveries: Le Duigou \& Knödlseder (2002) found 4 new cluster candidates in the Cygnus X area, and Ivanov et al. (2002) with an

Send offprint requests to: C. M. Dutra, e-mail: dutra@astro.iag.usp.br automated search in the 2MASS Second Release Point Source Catalogue found 6 new star clusters and candidates with respect to those reported in Bica et al.'s (2003) catalogue.

The present study aims at fulfilling the gap of detected IR star clusters in the Southern Milky Way by performing a search for IR star clusters and similar objects in the direction of known nebulae, using the recently available 2MASS all-sky release Atlas. In Sect. 2 we recall the previously catalogued clusters and stellar groups in this Milky Way Sector. In Sect. 3 we present the newly found objects related to optical and radio nebulae. In Sect. 4 we discuss some properties of the new samples. Finally, in Sect. 5 concluding remarks are given.

\section{Previously catalogued objects}

Several star clusters related to nebulae were previously known in the surveyed zone. For comparison purposes with the new objects (Sect. 3) we recall their properties in the following.

Twelve infrared clusters and stellar groups with $|b|<10^{\circ}$ are given in Bica et al. (2003), which are shown in Table 1. By Cols. (1) and (2) Galactic coordinates, (3) cluster or stellar group designation, (4) class (IRC - infrared star cluster or IRGr - infrared stellar Group), (5) major angular dimension, (6) distance from the Sun $R$, (7) multiplicity, (8) related nebulae, (9) cluster or stellar group major linear dimension. Angular dimensions correspond to limits where object still constrasts with respect the background, as estimated visually on $K$ or $K_{\mathrm{S}}$ images. Some additional distances with respect to Bica et al. (2003) are from studies given in Table 2 and Sect. 3. Multiplicity is a common phenomenon among infrared clusters (Bica et al. 2003): mP means member of pair, mT triplet, m5 quintuplet etc. 


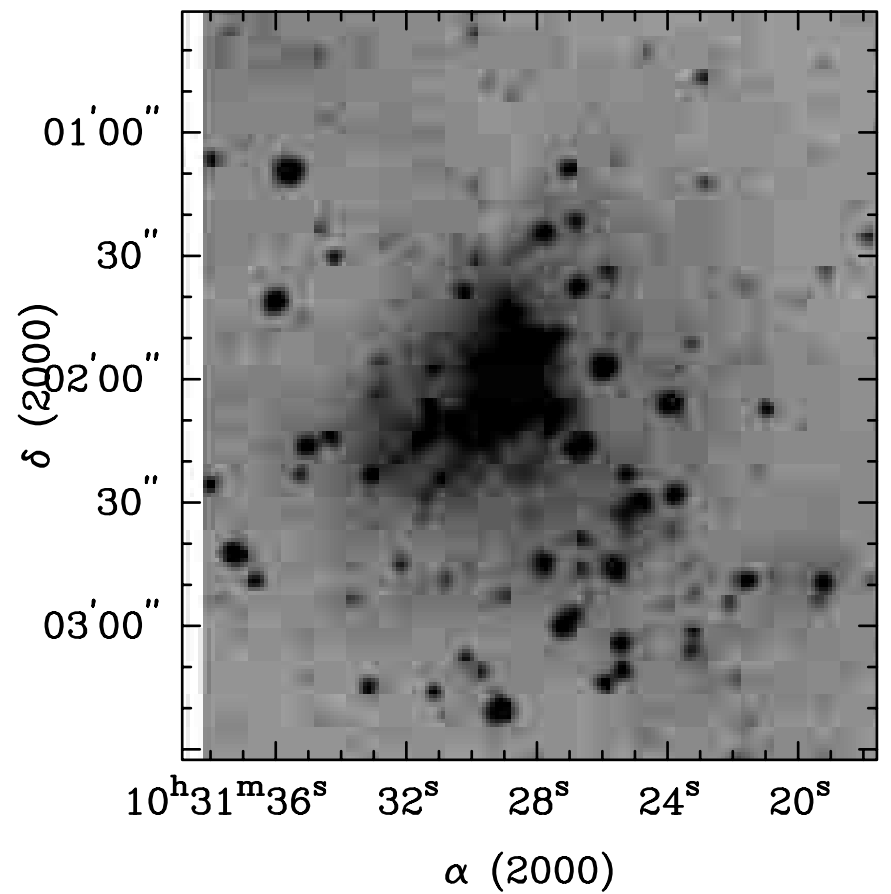

Fig. 1. $3^{\prime} \times 3^{\prime} 2$ MASS $K_{\mathrm{s}}$ image of prominent populous infrared cluster at $\mathrm{J} 2000 \alpha=10^{\mathrm{h}} 31^{\mathrm{m}} 29^{\mathrm{s}}$ and $\delta=-58^{\circ} 02^{\prime} 01^{\prime \prime}$ in the optical nebula Hoffleit $18=\mathrm{G} 285.3+0.0$.

Optical open cluster catalogues can be found in Alter et al. (1970), Lyngå (1987), and more recently in Dias et al. (2002). Thirty seven optical open clusters appear to be embedded in optical nebulae in the present sector, as shown in Table 2. By Cols. (1) and (2) Galactic coordinates, (3) optical angular size, (4) open cluster designation, (5) multiplicity, (6) related nebulae, (7) distance $R$, (8) age, (9) optical linear diameter, (10) class as judged from 2MASS images, (11) major angular dimension as measured on 2MASS images, and (12) corresponding linear dimension.

For the sake of homogeneity we measured the optical major angular dimension on first (DSS) and second generation (XDSS) digitized sky survey images using the Canadian Astronomy Data Centre extraction tool (http:// cadcwww.dao.nrc.ca/cadcbin/getdss). For larger angular size objects we used film copies of Schmidt plates from the ESO red (http://archive.eso.org/wdb/astrocat/ eso_schmidt.html) and UK blue (http://www.roe.ac. uk/ukstu/telescope.html) sky surveys. Pairs and multiplets are very common among embedded open clusters. Distances and ages are from the WEBDA database (Mermilliod 1996) - http://obswww.unige.ch/webda/, except those indicated in the table notes. The clusters are indeed young enough to be embedded or related to the nebular complexes. Cols. 10 to 12 will be discussed in Sect. 4.

\section{Newly found objects}

Since embedded clusters are expected to occur in the area of nebulae, we concentrated search efforts on known optical and radio nebulae, mostly HII regions but also reflection nebulae and supernova remnants.

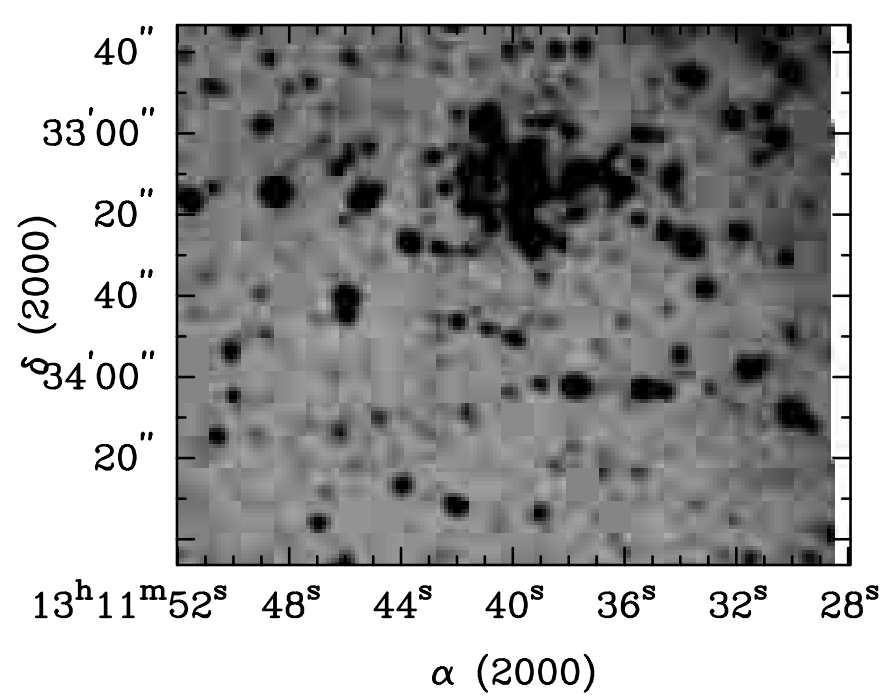

Fig. 2. $3^{\prime} \times 3^{\prime} 2$ MASS $K_{\mathrm{s}}$ image of prominent infrared cluster at J2000 $\alpha=13^{\mathrm{h}} 11^{\mathrm{m}} 39^{\mathrm{s}}$ and $\delta=-62^{\circ} 33^{\prime} 15^{\prime \prime}$ in the radio nebula $\mathrm{G} 305.3+0.2=$ AFGL4163.

The search employed the recently available 2MASS AllSky Release Images provided by means of the 2MASS Survey Visualization \& Image Server facility in the web interface http: //irsa.ipac. caltech. edu/. We extracted $J H K_{\mathrm{s}}$ images with $5^{\prime} \times 5^{\prime}$ centred on the coordinates of each nebula. For the nebulae with sizes larger than $5^{\prime} \times 5^{\prime}$ we took additional images of $10^{\prime} \times 10^{\prime}$ or $15^{\prime} \times 15^{\prime}$. The $K_{\mathrm{s}}$ band images allow one to probe deeper in more absorbed regions, and the $J$ and $H$ band images were used mostly as control of the presence of bright stars and as additional check for cluster resolvability. For the resulting IR star clusters we determined accurate positions and dimensions from their images in FITS format using SAOIMAGE 1.27.2 developed by Doug Mink. SAOIMAGE uses information in a 2MASS image header to transform linear to equatorial coordinates. Centers and angular dimensions are estimated visually on the 2MASS $K_{\mathrm{s}}$ images.

The optical nebula designations throughout this study are from Ced (Cederblad 1946), Hoffleit (Hoffleit 1953), Gum (Gum 1955), Sh2- (Sharpless 1959), RCW (Rodgers et al. 1960), GG (Georgelin \& Georgelin 1970a), GeGe (Georgelin \& Georgelin 1970b), vdBH-RN (van den Bergh \& Herbst 1975), ESO (Lauberts 1982), BFS (Blitz et al. 1982), Bran (Brand et al. 1986), BRC (Sugitani \& Ogura 1994). Some small angular size nebulae are from Wray (1966). The radio nebula $G$ designations are from various studies, namely Wilson et al. (1970), Caswell (1987) and Kuchar \& Clark (1997). MSH is from Mills et al. (1961) and references therein, and MHR from Mathewson et al. (1962). We also indicate some infrared nebulae related to sources in the AFGL and IRAS catalogues.

We merged the different catalogues and lists of nebulae into a radio/infrared and an optical nebula files. We cross-identified nebulae in each file, and then between the two files. Radio nebulae with optical counterparts were transferred to the optical file. We also used information of optical nebulae among radio detections by Caswell \& Haynes (1987). The resulting input lists of optical and radio nebulae contain respectively 991 and 276 objects in the present Milky Way sector, whose directions 
Table 1. Previously catalogued infrared clusters and stellar groups.

\begin{tabular}{|c|c|c|c|c|c|c|c|c|}
\hline$\overline{\bar{\ell}}$ & 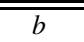 & Cluster or Stellar Group & Class & $\overline{\overline{D\left({ }^{\prime}\right)}}$ & $\overline{R(\mathrm{kpc})}$ & Mult. & Related nebulae & $\overline{L L D(\mathrm{pc})}$ \\
\hline 235.05 & -1.54 & IRAS07255-2012 Cluster & IRC & 1.1 & 10.2 & $\mathrm{mP}$ & in distant Molecular Cloud & 3.3 \\
\hline 235.06 & -1.55 & IRAS07255-2012 South Stellar Group & IRGr & 0.8 & 10.2 & $\mathrm{mP}$ & in distant Molecular Cloud & 2.4 \\
\hline 243.16 & 0.49 & NGC2467-East IR Cluster & IRC & 2.9 & 4.3 & m5 & in NGC2467 Neb.,in Gum9, in Sh2-311 & 3.4 \\
\hline 265.14 & 1.45 & RCW36 IR Cluster & IRC & 6.0 & 0.9 : & & in Gum20=RCW36=G265.1+1.5 & 1.6 \\
\hline 267.72 & -1.09 & vdBH-RN26 IR Cluster & IRC & 1.2 & 1.7 & $\mathrm{mP}$ & in vdB-RN26=BRC59, in RCW38 & 0.6 \\
\hline 267.92 & -1.06 & RCW38 IR Cluster & IRC & 2.0 & 1.7 & $\mathrm{mP}$ & in Gum22,in RCW38 & 1.0 \\
\hline 269.18 & -1.46 & Gum25 IR Cluster & IRC & 3.5 & 2.6 & & in Gum25=RCW40=G269.2-1.4 & 2.6 \\
\hline 287.81 & -0.82 & vdBH-RN43 IR Cluster & IRC & 1.5 & 2.5 & $\mathrm{~m} 10$ & in vdBH-RN43, in RCW53, in $\eta$ Car Complex & 1.1 \\
\hline 291.27 & -0.70 & NGC3576 IR Cluster & IRC & 2.0 & 2.4 & $\mathrm{mP}$ & rel NGC3581,in G291.284-0.713,in Gum38a=RCW57a & 1.4 \\
\hline 320.15 & 0.79 & RCW87 IR Cluster & IRC & 2.5 & 2.6 & $\mathrm{mP}$ & in RCW87,IRAS15015-5720,G320.2+0.8 & 1.9 \\
\hline 333.60 & -0.21 & G333.6-0.2 IR Cluster & IRC & 1.5 & 14.1 & & in G333.6-0.2 & 6.5 \\
\hline 336.48 & -1.48 & RCW108 IR Cluster & IRC & 0.9 & 1.3 & $\mathrm{mP}$ & in $\mathrm{BRC} 79=\mathrm{G} 336.5-1.5$,in RCW 108 & 0.34 \\
\hline
\end{tabular}

Table 2. Optical open clusters in nebulae.

\begin{tabular}{|c|c|c|c|c|c|c|c|c|c|c|c|}
\hline$\overline{\bar{\ell}}$ & $\bar{b}$ & $D_{(\prime)}$ & Open Cluster & Mult. & Related Nebulae & $R(\mathrm{kpc})$ & $t(\mathrm{Myr})$ & $L D(\mathrm{pc})$ & IR Class & IR $D\left({ }^{\prime}\right)$ & IR $L D(\mathrm{pc})$ \\
\hline 234.78 & -0.23 & 11 & Bochum 6 & $\mathrm{mP}$ & in Sh2-309=RCW13 & 4.0 & 10 & 13 & - & - & - \\
\hline 243.07 & 0.52 & 2.8 & Haf19 $9^{l}$ & m5 & in Sh2-311 & 5.1 & 8 & 4.2 & IRC & 1.5 & 2.2 \\
\hline 243.15 & 0.43 & 15 & $\mathrm{NGC} 2467^{l}$ & - & in NGC2467 Neb.,in Gum9, in Sh2-311 & $5.1^{k}$ & - & 22 & - & - & - \\
\hline 243.15 & 0.44 & 1.6 & Haf $18 \mathrm{a}^{l}$ & m5 & in Sh2-311, in NGC2467 & $6.0^{m}$ & $8^{m}$ & 2.8 & $\mathrm{IRC}^{g}$ & 1.2 & 2.1 \\
\hline 243.16 & 0.48 & 3 & Haf $18 \mathrm{c}^{l, m}$ & m5 & in Sh2-311, in NGC2467 & $6.0^{m}$ & $8^{m}$ & 5.2 & - & - & - \\
\hline 243.17 & 0.44 & 2.1 & Haf $18 b^{l, m}$ & m5 & in Sh2-311,in NGC2467 & $6.0^{k}$ & - & 3.8 & IRGr & 1.2 & 2.1 \\
\hline 261.45 & 0.99 & 17 & Cr197 & - & in Gum15=RCW32 & 0.8 & 13 & 4 & - & - & - \\
\hline 262.22 & -7.81 & 370 & Cr173 & - & in Gum Nebula & $0.4^{h}$ & $20^{h}$ & 43 & - & - & - \\
\hline 284.27 & -0.32 & 4 & Westerlund 2 & - & in Gum29, in RCW49 & 1.9 & $2.5^{i}$ & 2.2 & IRC & 2.2 & 1.2 \\
\hline 285.69 & 0.05 & 8 & Loden 153 & $\mathrm{mP}$ & in Gum30 & $2.3^{i}$ & & 6.0 & IROC & 4 & 2.7 \\
\hline 285.85 & 0.07 & 9 & NGC3293 & - & in Gum30 & 2.3 & $6^{i}$ & 5.4 & - & - & - \\
\hline 286.22 & -0.17 & 7 & IC2599 ${ }^{a}$ & - & in NGC3324 Neb. $=$ Gum31 $=$ G286.195-0.163 & $2.3^{k}$ & $6^{k}$ & 4.7 & $\mathrm{IRC}^{g}$ & 2.8 & 1.9 \\
\hline 286.24 & -0.16 & 14 & NGC3324 & - & in NGC3324 Neb. $=$ Gum31 $=$ G286.195-0.163 & 2.3 & 6 & 9.4 & - & - & - \\
\hline 286.76 & -1.65 & 20 & Bochum 9 & $\mathrm{~m} 10$ & in RCW53, in $\eta$ Car Complex & $2.7^{k}$ & - & 16 & - & - & - \\
\hline 287.02 & -0.31 & 23 & Bochum 10 & $\mathrm{~m} 10$ & in RCW53, in $\eta$ Car Complex & 2.0 & 7 & 13 & - & - & - \\
\hline 287.40 & -0.36 & 5.5 & $\operatorname{Tr} 15$ & $\mathrm{~m} 10$ & in RCW53, in $\eta$ Car Complex & 1.9 & 8 & 3.0 & $\mathrm{IRC}^{g}$ & 2.5 & 1.4 \\
\hline 287.41 & -0.57 & 8 & $\operatorname{Tr} 14$ & $\mathrm{~m} 10$ & in RCW53, in $\eta$ Car Complex & 2.7 & 7 & 6.3 & IRGr & 1.9 & 1.5 \\
\hline 287.49 & -0.53 & 7 & $\mathrm{Cr} 232$ & $\mathrm{~m} 10$ & in RCW53, in $\eta$ Car Complex & 3.0 & 5 & 5.5 & IRGr & 2.6 & 2.3 \\
\hline 287.55 & -1.02 & 29 & $\mathrm{Cr} 228$ & $\mathrm{~m} 10$ & in RCW53, in $\eta$ Car Complex & 2.2 & 7 & 6.1 & - & - & - \\
\hline 287.59 & -0.66 & 14 & $\operatorname{Tr} 16$ & $\mathrm{~m} 10$ & in RCW53, in $\eta$ Car Complex & 2.7 & 6 & 11 & - & - & - \\
\hline 287.64 & -0.68 & 4 & $\mathrm{Cr} 234$ & $\mathrm{~m} 10$ & in RCW53, in $\eta$ Car Complex & $2.7^{k}$ & - & 3.1 & - & - & - \\
\hline 288.03 & -0.86 & 4 & Bochum 11 & $\mathrm{~m} 10$ & in RCW53, in $\eta$ Car Complex & 2.4 & 6 & 2.8 & IRC & 1.7 & 1.2 \\
\hline 289.50 & 0.12 & 3 & Pis17 & - & in NGC3503=vdBH-RN46, in Gum34b,in RCW54 & 3.5 & $4.5^{i}$ & 3.1 & IRC & 1.5 & 1.5 \\
\hline 290.33 & -2.99 & 4 & Graham 1 & - & includes vdBH-RN45, in RCW55=G290.3-3.0 & $3.6^{f}$ & - & 4.2 & $\operatorname{IRC}^{g}$ & 2.1 & 2.2 \\
\hline 290.71 & 0.20 & 9 & NGC3572 & - & in Gum37=RCW54c & 2.0 & 8 & 5.2 & - & - & - \\
\hline 291.61 & -0.52 & 1.9 & NGC3603 & - & in Gum $38 b=$ RCW $57 b$ & 3.6 & $2^{i}$ & 2.0 & IRC & 1.8 & \\
\hline 294.81 & -1.64 & 11 & IC2944 & $\mathrm{mP}$ & in Gum42=RCW62 & 1.8 & 7 & 5.8 & - & - & - \\
\hline 294.96 & -1.71 & 11 & IC2948 & $\mathrm{mP}$ & in Gum42=RCW62 & $1.8^{k}$ & - & 5.8 & - & - & - \\
\hline 306.07 & 0.20 & 4.5 & Stk16nw ${ }^{b}$ & $\mathrm{mP}$ & in RCW75=Gum48a & 1.9 & 5 & 2.5 & - & - & - \\
\hline 306.10 & 0.12 & 2 & Stk16se $e^{c}$ & $\mathrm{mP}$ & in vdBH-RN60a, in RCW75=Gum48a & $1.9^{k}$ & & 1.1 & IRGr & 1.4 & 0.8 \\
\hline 308.67 & 0.60 & 1.5 & $\mathrm{BH} 151^{d}$ & - & in RCW79 & $3.8^{j}$ & - & 1.7 & IRC & 1.2 & 1.3 \\
\hline 320.51 & -1.20 & 4.5 & Pis 20 & - & rel. to radio SNR MSH15-52e & 2.0 & 7 & 2.6 & IRC & 3.0 & 1.7 \\
\hline 336.71 & -1.59 & 16 & NGC6193 & $\mathrm{mP}$ & in RCW108=Gum53 & 1.2 & 6 & 5.6 & IROC & 7.0 & 2.1 \\
\hline 343.46 & 1.17 & 17 & NGC6231 & $\mathrm{m} 4$ & in Gum55=RCW113 & 1.2 & 7 & 5.9 & IROC & 6.5 & 2.3 \\
\hline 344.39 & 1.66 & 55 & Harvard 12 & $\mathrm{~m} 4$ & in Gum55=RCW113 & 1.2 & - & 19 & - & - & - \\
\hline 344.60 & 1.61 & 5 & $\mathrm{BH} 205^{d}$ & $\mathrm{~m} 4$ & in Gum55=RCW113 & 1.2 & - & 1.7 & IROC & 5 & 1.7 \\
\hline 344.94 & 1.60 & 45 & $\operatorname{Tr} 24$ & $\mathrm{~m} 4$ & in Gum55=RCW113 & 1.1 & 8 & 14 & - & - & - \\
\hline
\end{tabular}

Notes: ${ }^{a}$ central part of NGC 3324 open cluster, which is the object in WEBDA; ${ }^{b}$ Stk 16 in WEBDA; ${ }^{c}$ Stk 16 corresponding to the original coordinates (Alter et al. 1970); ${ }^{d}$ van den Bergh \& Hagen (1975); ${ }^{e}$ optical filaments are RCW 89; ${ }^{f}$ from Graham (1970); ${ }^{g}$ loose IRC; ${ }^{h}$ adopted from Hipparcos; ${ }^{i}$ adopted from Piatti et al. (2002); ${ }^{j}$ adopted from the nebula complex (Sect. 3); ${ }^{k}$ adopted from other cluster in the complex; ${ }^{l}$ NGC 2467 includes Haf 19, Haf 18a,b,c; NGC 2467 in WEBDA corresponds to a foreground group of stars; ${ }^{m}$ Haf 18a and Haf $18 \mathrm{~b}$ are respectively the north and south parts of Haf 18 in WEBDA.

were inspected. The whole Milky Way radio and optical nebula catalogue currently has 4454 entries after cross-identifications, and will be provided in a forthcoming study. It follows similar procedures as those used in the construction of the dark nebula catalogue (Dutra \& Bica 2002) which has 5004 entries.
The results of the cluster survey will be only available as Tables 3 and 4 in electronic form at CDS (Strasbourg) via anonymous ftp to cdsarc.u-strasbg.fr (130.79.128.5) or via

http://cdsweb.u-strasbg.fr/cgi-bin/qcat?/A+A/400/533, 


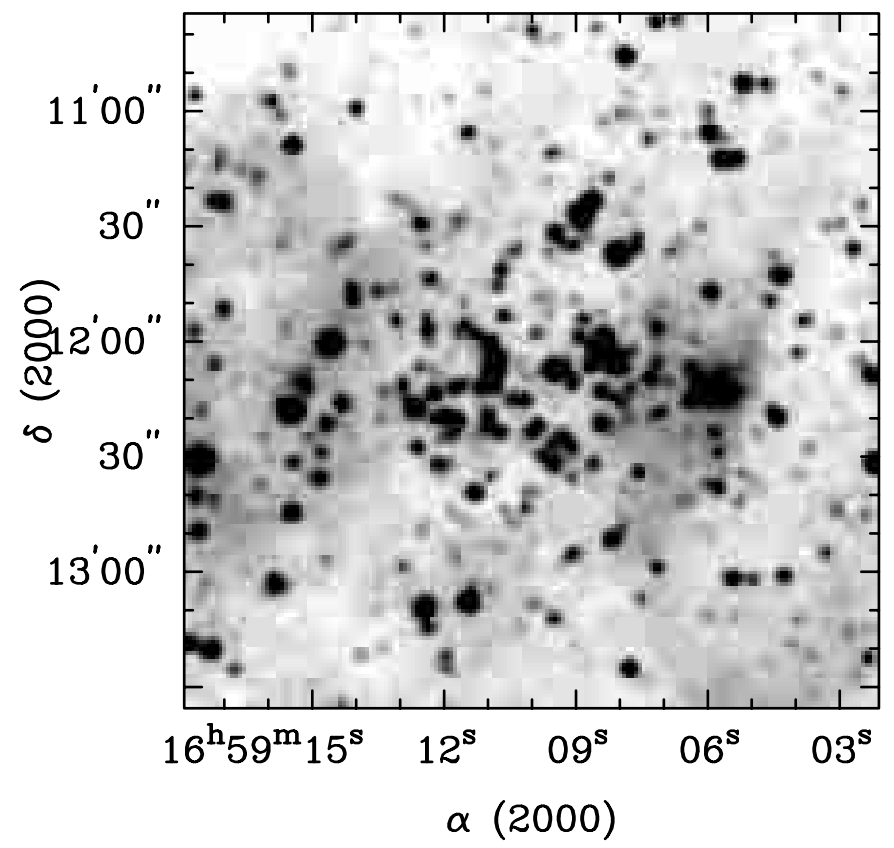

Fig. 3. $3^{\prime} \times 3^{\prime} 2$ MASS $K_{\mathrm{s}}$ image of loose infrared cluster at J2000 $\alpha=16^{\mathrm{h}} 59^{\mathrm{m}} 10^{\mathrm{s}}$ and $\delta=-40^{\circ} 12^{\prime} 05^{\prime \prime}$ in the nebula G345.3+1.5.

respectively for optical and radio nebulae. By Cols. (1) running number; (2) and (3) Galactic coordinates, (4) and (5) J2000.0 equatorial coordinates, (6) and (7) major and minor angular dimensions, (8) related nebulae, (9) class, (10) remarks including distance $R$ (in case of kinematical ambiguity the near and far distances are shown), multiplicity and linear dimension. The new infrared clusters, stellar groups and candidates from the optical nebula survey amount to 123 , and from the radio nebula survey 56. The rates of detection relative to the input nebula catalogues are 12 and $20 \%$ for optical and radio nebulae, respectively. The higher detection rate among radio nebulae is surely because they represent more often overall complexes, while optical nebulae deal more often with structural details of closer complexes.

Object classes are infrared cluster (IRC), stellar group (IRGr), cluster candidate (IRCC), and open cluster (IROC). IRCs are in general populous and at least partially resolved. We illustrate in Fig. 1 a prominent compact IRC (Object 48) related to an optical nebula, and in Fig. 2 one (Object 131) related to a radio nebula. A loose IRC (Object 114) is shown Fig. 3. A deeply embedded in nebular emission and/or reflection partially resolved IRC (Object 33) is given in Fig. 4. This object has a clear dust lane, and remarkably resembles the wellknown NGC 2024 cluster (e.g. Lada et al. 1991). The infrared cluster (Object 146) in the radio nebula G327.3-0.5 is also similar to that in NGC 2024. IRCCs are probably clusters, but are essentially unresolved, and require higher resolution and deeper images for definitive diagnostic (e.g. Object 8 - Fig. 5). IRGrs are less dense than IRCs, some are rather compact but little populated. An IRGr (Object 20) is given in Fig. 6. IROCs (e.g. Object 61 - Fig. 7) have similar appearence to optical open clusters and relatively large angular size $\left(\approx 2^{\prime}\right.$ or more). In Table 3 there occur 46 IRC, 64 IRGr, 7 IRCC and 6 IROC

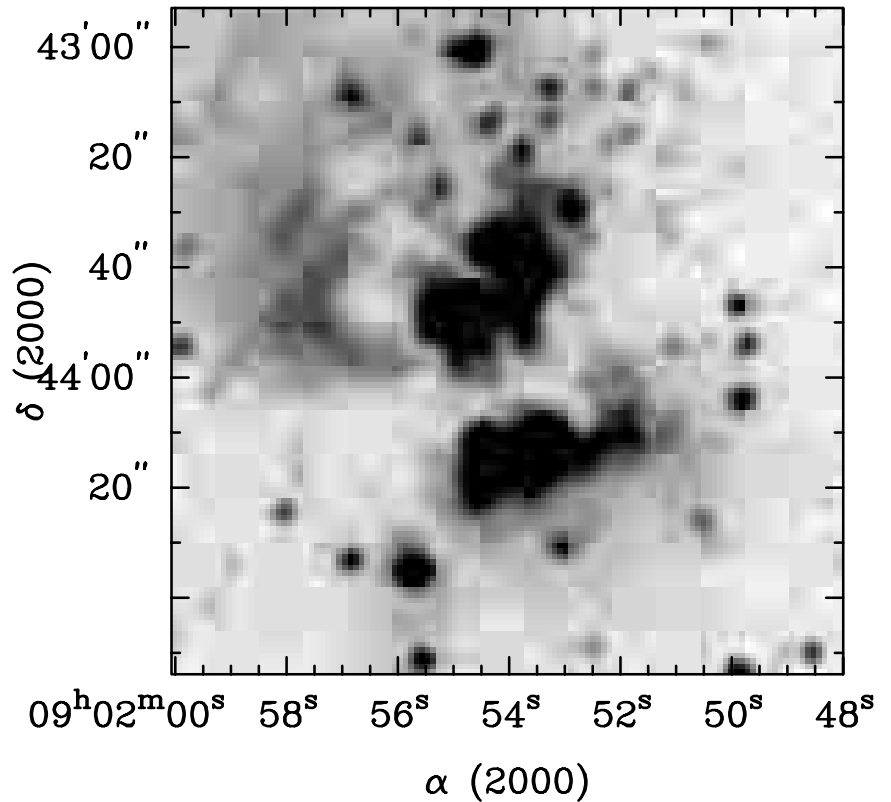

Fig. 4. $2^{\prime} \times 2^{\prime} 2$ MASS $K_{\mathrm{s}}$ image of deeply embedded cluster at J2000 $\alpha=9^{\mathrm{h}} 01^{\mathrm{m}} 54^{\mathrm{s}}$ and $\delta=-47^{\circ} 43^{\prime} 53^{\prime \prime} \mathrm{J} 2000$ in the nebula Bran 222 . The object is remarkably similar to the well-known infrared cluster in NGC 2024.

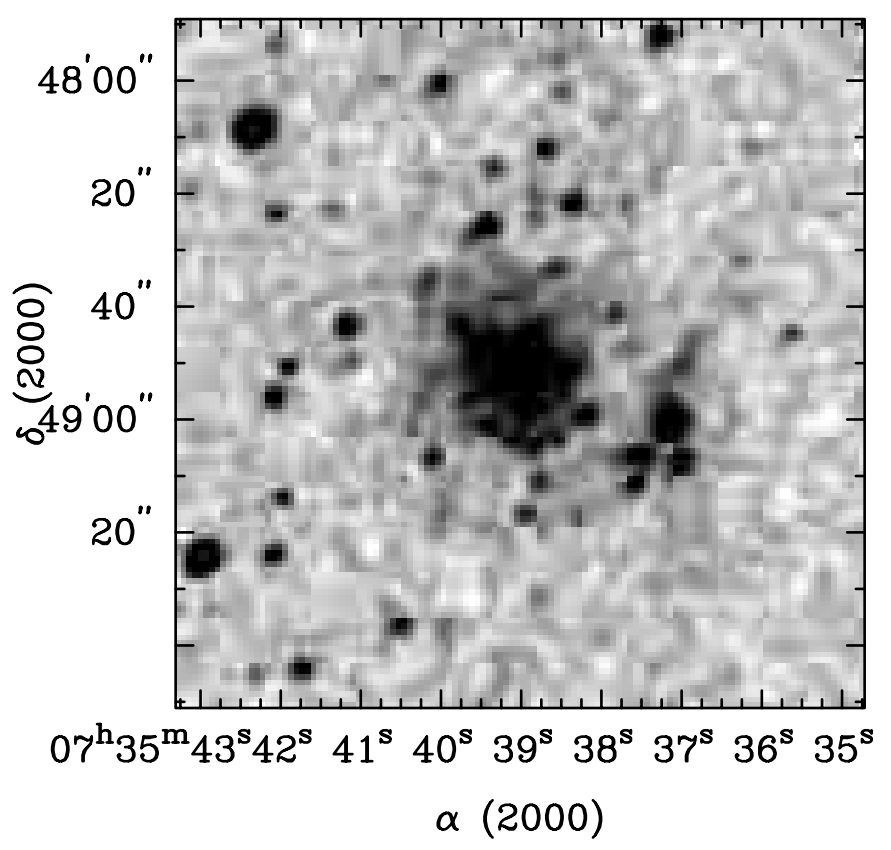

Fig. 5. $2^{\prime} \times 2^{\prime}$ 2MASS $K_{\mathrm{s}}$ image of infrared cluster candidate at $\mathrm{J} 2000 \alpha=07^{\mathrm{h}} 35^{\mathrm{m}} 39^{\mathrm{s}}$ and $\delta=-18^{\circ} 48^{\prime} 50^{\prime \prime}$ in the nebula Gum $7=$ Sh 2-307 = RCW 12 .

objects, while in Table 431 IRC, 8 IRGr, 16 IRCC and 1 IROC objects.

Distances are mostly based on kinematical estimates for the nebulae (Wilson et al. 1970; Caswell \& Haynes 1987), but include as well averages with estimates from individual stars, when available (e.g. Georgelin et al. 1973; Georgelin et al. 2000 and references therein). The near/far distance ambiguity has been solved by some of these authors $(R)$, else we indicate both $\left(R_{\mathrm{n}}\right.$ or $\left.R_{\mathrm{f}}\right)$. 


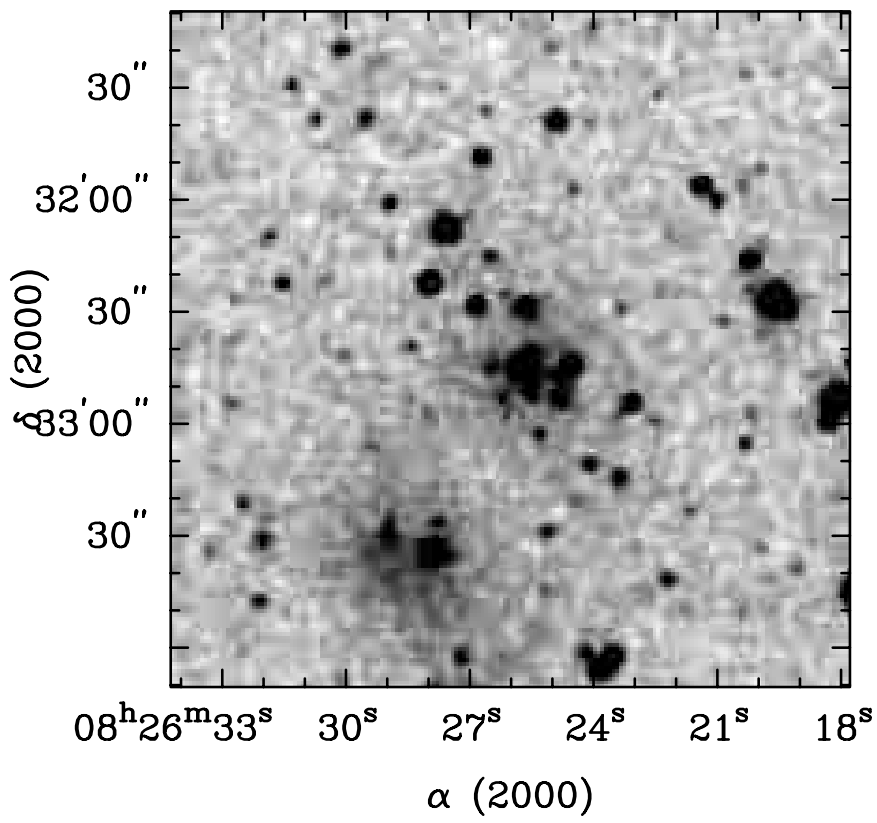

Fig. 6. $3^{\prime} \times 3^{\prime} 2$ MASS $K_{\mathrm{s}}$ image of infrared stellar group at J2000 $\alpha=8^{\mathrm{h}} 26^{\mathrm{m}} 26^{\mathrm{s}}$ and $\delta=-42^{\circ} 32^{\prime} 40^{\prime \prime}$ in the nebula Bran 149 .

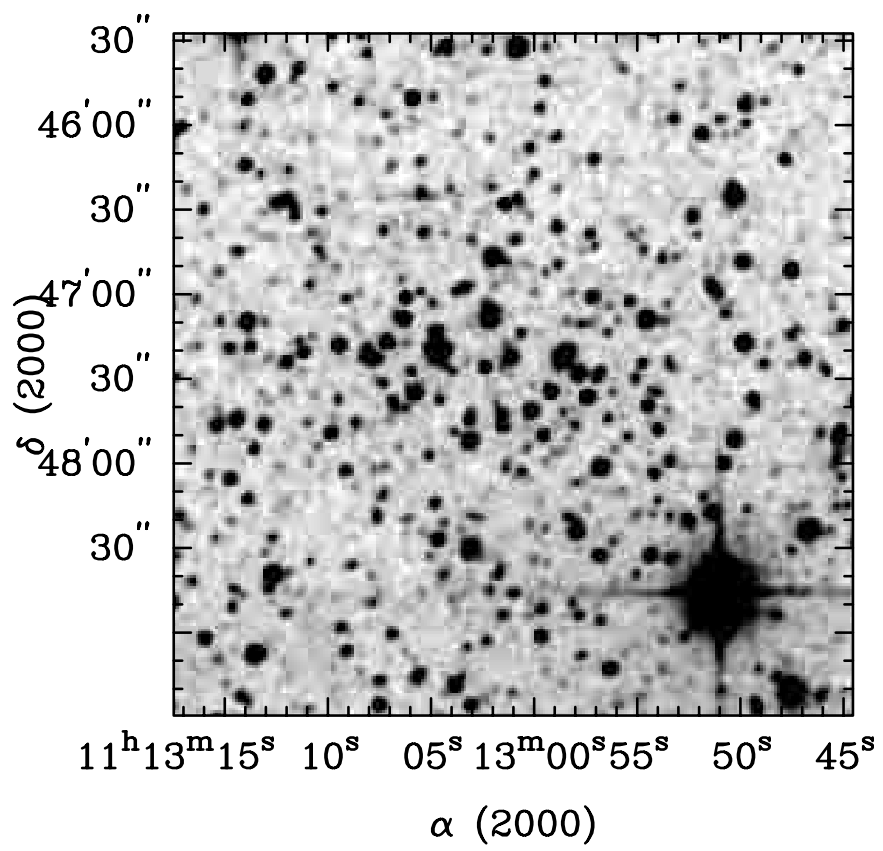

Fig. 7. $4^{\prime} \times 4^{\prime} 2$ MASS $K_{\mathrm{s}}$ image of infrared cluster at J2000 $\alpha=$ $11^{\mathrm{h}} 13^{\mathrm{m}} 01^{\mathrm{s}}$ and $\delta=-60^{\circ} 47^{\prime} 28^{\prime \prime}$ with appearence of typical optical open cluster in the nebula GG $2=$ G291.2-0.2.

One prominent cluster (Object 23) appears to be related to the optical nebula Munch 23 (Munch 1955), which is thus not a planetary nebula (K2-15 or PNG263.2+00.4, Acker 1992). Likewise, the nebulae ESO $313 * \mathrm{~N} 10$ and ESO 128EN25 are not planetary nebulae.

We included in Table 3 new embedded clusters and stellar groups related to optical reflection nebulae. This object type has been discussed in Dutra \& Bica (2001). They appear to be less massive clusters or stellar groups where no ionizing star was formed. The objects in Soares \& Bica (2002) are of this

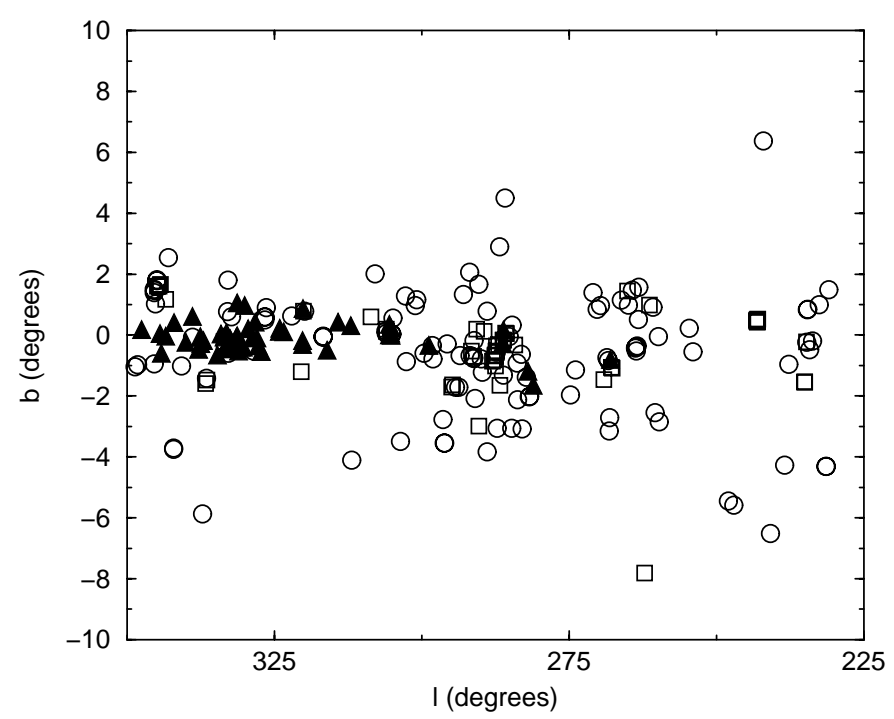

Fig. 8. Angular distribution of infrared clusters found in the vicinity of optical nebulae (Table 3) represented by open circles, and radio nebulae (Table 4) represented by filled triangles. Known star clusters in the region (Tables 1 and 2) are the open squares.

type or close to its limit towards ionizing stars. Detected objects in the van den Bergh \& Herbst (1975) reflection nebulae are probably of this type.

The Gum 38a complex (often designated NGC 3576 H II region) is well-known for harbouring the prominent NGC 3576 IR cluster (e.g. Persi et al. 1994). However, we realized that it is not related to the original NGC catalogue small nebula NGC 3576 (Table 1). The massive cluster is related to the radio H II region G291.284-0.713, and its optical counterpart NGC 3581. The small nebula NGC 3576 itself has a less massive cluster (Object 65 - Table 3), thus forming a cluster pair in that complex. Owing to the usual NGC 3576 designation in the literature for the massive cluster, we suggest NGC 3576A for the small one.

Some of the BRC nebulae (bright-rimmed clouds) have been shown to be sites of star cluster formation (Sugitani et al. 1995). The present study indicates 4 BRC clouds with new clusters or stellar groups (Table 3), together with 2 BRCs in Table 1 .

\section{Discussion}

We compare in Fig. 8 the angular distributions in galactic coordinates of the present samples (Tables 3 and 4) with those of previously known infrared objects (Table 1) and the optical open clusters (Table 2). The increase of the sample of known clusters and stellar groups is overwhelming. Note that the infrared clusters coming from the radio nebulae are mainly located between $300^{\circ}<\ell<350^{\circ}$, corresponding to internal arms and where absorption in the Galaxy shows a pronounced increase (Dutra \& Bica 2000b).

The distance histograms for the objects obtained from the optical and radio nebula samples (Tables 3 and 4, respectively) are shown in Fig. 9. For the objects with distance ambiguity we assumed their near distance as a lower limit for the 

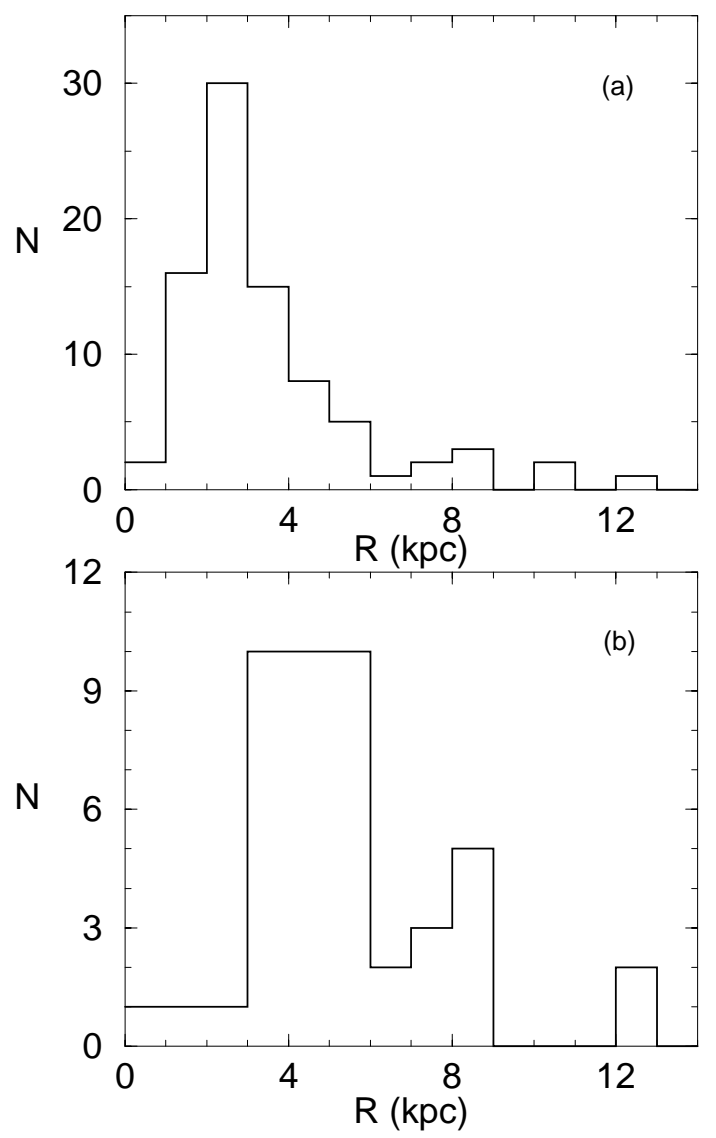

Fig. 9. Distance histograms for the samples coming from: panel (a) optical (Table 3) and panel (b) radio nebulae (Table 4).

histogram analysis. The histograms show that objects coming from radio nebulae are on the average more distant peaking at $\approx 4.5 \mathrm{kpc}$, while those from optical nebulae peak at $\approx 2.5 \mathrm{kpc}$. We conclude that a typical embedded cluster discovered with 2MASS is not very far in the Galaxy, but the sampled clusters are located in more internal arms than Sagittarius-Carina, like Scutum-Crux and beyond (e.g. Georgelin \& Georgelin 1976).

The linear size histograms for the objects obtained from the optical and radio nebula samples (Tables 3 and 4, respectively) are shown in Fig. 10. The objects with distance ambiguity were excluded. The distributions are similar to that obtained in Bica et al. (2003). A typical infrared cluster is small (less than $3 \mathrm{pc}$ in linear diameter). The peak for the objects from the radio nebulae is shifted to larger sizes, suggesting that on the average they are more massive clusters. At such young ages, star clusters are not dynamically relaxed and their dimensions are not yet modulated by the Galactic tidal field. Consequently, sizes must reflect formation conditions.

An important issue concerns the properties of the optical open clusters (Table 2), as compared to those of the IR objects (Tables 1, 3 and 4), if measured with the same material. Out of 36 optical open clusters, 17 (Table 2) could be retrieved with the same procedures that we used to probe the new IR clusters. The sizes for the retrieved optical open clusters measured on 2MASS material with the present procedures are systematically smaller in the infrared than in the optical (compare
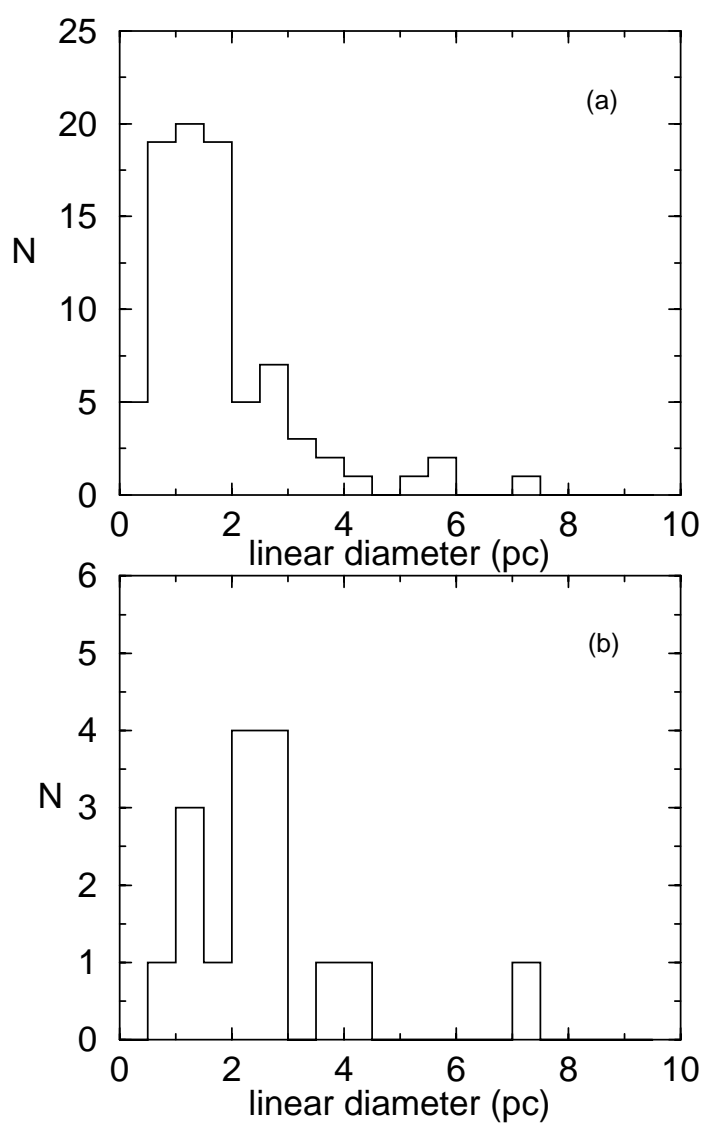

Fig. 10. Linear major dimension histogram for the samples coming from: panel a) optical (Table 3) and panel b) radio nebulae (Table 4).

Cols. 8 and 11 of Table 2), suggesting that we are preferentially seeing denser parts and cluster cores in the infrared. Probably the optical nebula complex Gum $55=\mathrm{RCW} 113$, owing to the low absorption and proximity, can clarify the issue on stellar component structure in a complex after a few Myr (Table 2). It contains the populous cluster NGC 6231 with an optical diameter of $\approx 6 \mathrm{pc}$ and the loose cluster BH 205 with $\approx 2 \mathrm{pc}$, embedded in the extended stellar aggregates Harvard 12 and $\operatorname{Tr}$ 24, with 19 and $14 \mathrm{pc}$, respectively.

No prominent cluster was found to be related to radio SNRs. A probable IRGr is in the area of the radio SNR G330.2+1.0, and one IRCC is in that of G323.5+0.1 (Table 4). Among optical open clusters the association to SNRs is a rare phenomenon too: only Pis 20 appears to have one (Table 2).

Some infrared clusters and stellar groups occur in pairs or triplets in a fraction of $25 \%$ (Bica et al. 2003). For the present sample (Tables 3 and 4) we obtain $21 \%$, somewhat lower but suggesting as well a significant role of multiplicy on cluster formation and early dynamical evolution. Taking only the clusters of Table 3, which are closer on the average (Fig. 9), the resulting fraction is $26 \%$. For the objects in Table 4 the fraction is $9 \%$. Small companions would be more difficult to detect in highly absorbed zones and/or for more distant clusters. 


\section{Concluding remarks}

We searched for embedded star clusters in the directions of 991 optical and 276 radio nebulae in the Southern Milky Way (encompassing the region $|b|<10^{\circ}, 230^{\circ}<\ell<350^{\circ}$ ) using 2MASS all-sky release $J H K_{\mathrm{s}}$ images. A total of 179 new infrared clusters, stellar groups, candidates and open clusters were found. They are interesting targets for detailed future infrared studies. The sample of known infrared clusters and similar objects in the Galaxy is now increased by $63 \%$, as compared to 276 objects in Bica et al.'s (2003) infrared catalogue together with 10 recent additions (Le Duigou \& Knödlseder 2002; Ivanov et al. 2002).

Assuming that the new infrared clusters and stellar groups are physically linked to the optical or radio nebulae, we estimated distances and linear diameters for most of them. On the average objects coming from radio nebulae are more distant (distribution peak at $4.5 \mathrm{kpc}$ ) than those from optical nebulae (peak at $2.5 \mathrm{kpc}$ ). The linear diameter distributions indicate that objects from the radio nebulae are typically somewhat larger than those from optical nebulae, suggesting that they are in general clusters in more massive complexes.

In the studied Southern Milky Way sector we detected embedded clusters/stellar groups related to 12 and $20 \%$ of the catalogued optical and radio nebulae, respectively. Considering these detection rates, the remaining nebulae in the disc and the fact that other Milky Way sectors have been more surveyed (Bica et al. 2003), one can infer that about 200 infrared star clusters might still be found with the 2MASS Atlas using the present method. In addition to that search, non-embedded infrared clusters should be surveyed by means of systematic visual inspections and/or automated methods, for a nearly complete census of infrared clusters.

Acknowledgements. This publication makes use of data products from the Two Micron All Sky Survey, which is a joint project of the University of Massachusetts and the Infrared Processing and Analysis Center/California Institute of Technology, funded by the National Aeronautics and Space Administration and the National Science Foundation. We employed catalogues from CDS/Simbad (Strasbourg) and Digitized Sky Survey images from the Space Telescope Science Institute (U.S. Government grant NAG W-2166) obtained using the extraction tool from CADC (Canada). We also made use of the WEBDA open cluster database. We acknowledge support from the Brazilian Institutions CNPq and FAPESP. CMD acknowledges FAPESP for a post-doc fellowship (proc. 00/11864-6).

\section{References}

Acker, A., Ochsenbein, F., Stenholm, B., et al. 1992, Strasbourg-ESO Catalogue of Galactic Planetary Nebulae, CDS, Strasbourg

Alter, G., Ruprecht, J., \& Vanysek, V. 1970, Catalogue of star clusters and associations + supplements, 2nd edn. (Akad. Kiado, Budapest)

Bica, E., Dutra, C. M., \& Barbuy, B. 2003, A\&A, 397, 177

Blitz, L., Fich, M., \& Stark, A. A. 1982, ApJS, 49, 183

Brand, J., Blitz, L., \& Wouterloot, J. G. A. 1986, A\&AS, 65, 537
Carpenter, J. M. 2000, AJ, 120, 3139

Caswell, J. I. 1987, A\&A, 171, 261

Cederblad, S. 1946, MeLu2, 119, 1

Deharveng, L., Zavagno, A., Cruz-González, I., et al. 1997, A\&A, 317,459

Dias, W. S., Alessi, B. S., Moitinho, A., \& Lépine, J. R. D. 2002, A\&A, 389, 871

Dutra, C. M., \& Bica, E. 2000a, A\&A, 359, L9

Dutra, C. M., \& Bica, E. 2000b, A\&A, 359, 347

Dutra, C. M., \& Bica, E. 2001, A\&A, 376, 434

Dutra, C. M., \& Bica, E. 2002, A\&A, 383, 631

Epchtein, N., de Batz, B., Capoani, L., et al. 1997, The Messenger, 87, 27

Georgelin, Y. P., \& Georgelin, Y. M. 1970a, A\&A, 7, 133

Georgelin, Y. P., \& Georgelin, Y. M. 1970b, A\&AS, 3, 1

Georgelin, Y. M., \& Georgelin, Y. P. 1976, A\&A, 49, 57

Georgelin, Y. M., Russell, D., Amram, P., et al. 2000, A\&A, 357, 308

Georgelin, Y. M., Georgelin, Y. P., \& Roux, S. 1973, A\&A, 25, 337

Graham, J. A. 1970, AJ, 75, 703

Gum, C. S. 1955, MmRAS, 67, 155

Hodapp, K.-W. 1994, ApJS, 94, 615

Hoffleit, D. 1953, AnHar, 119, 37

Hurt, R. L., Jarrett, T. H., Kirkpatrick, J. D., \& Cutri, R. M. 2000, AJ, 120,1876

Kuchar, T. A., \& Clark, F. O. 1997, ApJ, 488, 224

Ivanov, V. D., Borissova, J., Pessev, P., Ivanov, G. R., \& Kurtev, R. 2002, A\&A, 394, L1

Lada, E. A., Evans, N. J. II, Depoy, D. L., \& Gatley, I. 1991, ApJ, 371, 171

Lada, C. J., \& Lada, E. A. 1991, in The formation and evolution of star clusters, 3

Lauberts, A. 1982, ESO/Uppsala survey of the ESO(B) atlas, Garching: ESO

Lyngå, G. 1987, Computer based catalogue of open cluster data, 5th edn. (Strasbourg: CDS)

Le Duigou, J.-M., \& Knödlseder, J. 2002, A\&A, 392, 869

Mathewson, D. S., Healey, J. R., \& Rome, J. M. 1962, AuJPh, 15, 354

Mermilliod, J. C. 1996, in The origins, evolution, and destinies of binary stars in clusters, ASP Conf. Ser., 90, 475

Mills, B. Y., Slee, O. B., \& Hill, E. R. 1961, AuJPh, 14, 497

Munch, L. 1955, BOTT, 13, 28

Piatti, A. E., Bica, E., Clariá, J. J., Santos, J. F. C., \& Ahumada, A. V. 2002, MNRAS, 335, 233

Reylé, C., \& Robin, A. C. 2002, A\&A, 384, 403

Rodgers, A. W., Campbell, C. T., \& Whiteoak, J. B. 1960, MNRAS, 121,103

Schlegel, D. J., Finkbeiner, D. P., \& Davis, M. 1998, ApJ, 500, 525

Soares, J. B., \& Bica, E. 2002, A\&A, 388, 172

Sharpless, S. 1959, ApJS, 4, 257

Skrutskie, M., Schneider, S. E., Stiening, R., et al. 1997, in The Impact of Large Scale Near-IR Sky Surveys, ed. Garzon et al. (Netherlands: Kluwer), 210, 187

Sugitani, K., \& Ogura, K. 1994, ApJS, 92, 163

Sugitani, K., Tamura, M., \& Ogura, K. 1995, ApJ, 455, L39

van den Bergh, S., \& Herbst, W. 1975, AJ, 80, 208

van de Bergh, S., \& Hagen, G. L. 1975, AJ, 80, 11

Wilson, T. L., Mezger, P. G., Gardener, F. F., \& Milne, D. K. 1970, A\&A, 364, 384

Wray, J. D. 1966, Thesis, Northwestern Univ., Illinois, USA 